\title{
Reporte del primer caso de cistoadenoma papilar de epididimo en Colombia, Hospital Universitario de Santander
}

\author{
Gabriel Pérez-García* \\ María Paula Pérez-Barón**
}

\begin{abstract}
* Médico Patólogo. Profesor Asociado y Coordinador de Posgrado de Patología. Departamento de Patología. Grupo de Patología Estructural. Funcional y Clínica de la Universidad Industrial de Santander (PAT-UIS). Facultad de Salud, Universidad Industrial de Santander, Bucaramanga. Colombia. **Médica Servicio Social Obligatorio. E.S.E. Hospital Regional de Chiquinquirá. Boyacá. Colombia.

Correspondencia: Dr. Gabriel Eduardo Pérez García. Departamento de Patología, Universidad Industrial de Santander, Facultad de Salud, Carrera 32 No. 29-31, Bucaramanga, Santander, Colombia. Teléfono: (+57) 3185320299. Correo electrónico: geperezg@uis.edu.co
\end{abstract}

Resumen

La presencia de una masa en la región escrotal comprende un amplio espectro de patologías, unas con mayor urgencia para el manejo que otras, dado el riesgo asociado de pérdida testicular o desarrollo de cáncer. Dentro de las posibilidades diagnósticas se encuentran las neoplasias, destacando las originadas en el epidídimo, con un $75 \%$ de casos de etiología benigna, correspondiendo un $9 \%$ al cistoadenoma papilar de epidídimo que sobresale por su baja frecuencia dado que en la literatura mundial existen aproximadamente 70 reportes publicados con escasos provenientes de literatura médica en castellano, en los que se incluye el presente caso expuesto en el que se confirma histopatológicamente un cistoadenoma papilar de epidídimo y se presenta una revisión del tema acerca del enfoque de un paciente con masa escrotal. Asimismo se resalta el hecho de que esta lesión puede asociarse con la enfermedad de Von Hippel Lindau, sobre todo en presentaciones bilaterales. MÉD.UIS. 2020;33(2):95-100.

Palabras clave: Cistoadenoma papilar. Epidídimo. Enfermedades de los Genitales Masculinos. Neoplasias.

\section{Report of the first case of papillary cystadenoma of the epididymis in Colombia}

Abstract

The presence of a mass in the scrotal region comprises a wide spectrum of pathologies, some with greater urgency for management than others, given the associated risk of testicular loss or cancer development. Among the diagnostic possibilities are the neoplasms, highlighting those originating in the epididymis, with $75 \%$ of cases of benign etiology, $9 \%$ corresponding to the papillary cystadenoma of the epididymis that stands out for its low frequency given that in the world literature there are approximately 70 published reports with few from medical literature in Spanish, which include the present case presented in which a papillary cystadenoma of the epididymis is confirmed histopathologically and a review of the topic about the approach of a patient with scrotal mass is presented. Also the fact that this lesion can be associated with von Hippel Lindau disease, especially in bilateral presentations is highlighted. MÉD.UIS. 2020;33(2):95100.

Keywords: Papillary cystadenoma. Epididymis. Genital Diseases, Male. Neoplasms

¿Cómo citar este artículo?: Pérez-García G, Pérez-Barón MP. Reporte del primer caso de cistoadenoma papilar de epidídimo en Colombia, Hospital Universitario de Santander. MÉD.UIS. 2020;33(2):95-100. doi: 10.18273/revmed.v33n2-2020011 


\section{Introducción}

Las neoplasias del epidídimo son raras, alrededor de un $75 \%$ son de etiología benigna, la más común es el tumor adenomatoide, seguido por el cistoadenoma papilar ${ }^{1,2}$. Por otro lado, los tumores malignos muestran tasas de incidencia de aproximadamente $0,03 \%$ en los que se incluye el adenocarcinoma, mesotelioma y carcinoma de células renales metastásico ${ }^{2,3}$. Dentro del grupo de neoplasias benignas del epidídimo se destaca el cistoadenoma papilar que corresponde al $9 \%$ del total de tumores, es de origen epitelial y se ha reportado en diversas publicaciones; en la literatura mundial existen aproximadamente 70 reportes publicados con escasos provenientes de literatura médica en castellano, es una patología infrecuente que debe tenerse en cuenta como diagnóstico diferencial en el contexto de un paciente con masa escrotal ${ }^{2-3,5-6}$.

El cistoadenoma papilar de epidídimo suele presentarse principalmente en adultos jóvenes, con un rango de expresión que va desde los 16 hasta los 76 años, con un promedio de 35 años, se caracteriza por ser una lesión sólido quística de crecimiento lento, que puede medir de 1 a $3 \mathrm{~cm}$, se ubica principalmente en la región de la cabeza del epidídimo, y es generalmente asintomática pero puede producir un cuadro de edema escrotal secundario, es usualmente indoloro, aunque la mayoría de veces se encuentra como hallazgo incidental durante un examen físico por cualquier otra razón ${ }^{3-5}$. El diagnóstico es inicialmente clínico y con ecografía testicular, el manejo consiste en la escisión local con preservación testicular, aunque en algunos casos pueden requerir orquiectomía ${ }^{4}$. Se resalta que el hallazgo de cistoadenoma papilar de epidídimo bilateral es patognomónico de la enfermedad de Von Hippel Lindau, un síndrome con alto riesgo de desarrollar neoplasias benignas y malignas por lo que los pacientes deben continuar, en dichos casos, un tamizaje radiológico o genético ${ }^{1,4}$.

Los objetivos de este artículo incluyen reportar el primer caso diagnosticado en el Hospital Universitario de Santander, Colombia, de un cistoadenoma papilar de epidídimo, y de igual forma revisar el espectro de presentaciones que puede llegar a tener un paciente que ingresa para estudio de una masa escrotal y su amplia gama de diagnósticos probables.

\section{Presentación del caso}

Masculino de 26 años remitido del servicio de consulta externa de urología del Hospital Universitario de Santander por haber presentado una masa en región escrotal derecha de un mes de evolución asociada a dolor e incomodidad. El paciente llegó acompañado de familiar, quien suministró la mayor parte de la información, dado que el paciente tenía disartria y disminución en la concentración, amaurosis con estrabismo en ojo derecho con atrofia del nervio óptico y disminución de la agudeza visual del ojo izquierdo como secuelas secundarias al antecedente personal de hemangioblastoma cerebeloso localizado en región paramediana izquierda para lo que fue manejado con resección quirúrgica y colocación de una válvula de Hakim.

Al examen físico se encuentra paciente longilíneo, signos vitales normoconfigurados, con evidente alteración en el lenguaje y en la atención que dificultaban la comunicación con el paciente. A la exploración genital en la inspección no se observaban cambios inflamatorios en la piel, no presentaba asimetría de las bolsas escrotales, en la palpación de sacos escrotales se evidenciaba en la región lateral del saco escrotal derecho una masa móvil, renitente, dolorosa a la palpación, no adherida a planos profundos, no se palpaban adenopatías asociadas a la lesión.

Los paraclínicos de ingreso evidenciaron hemograma con leucocitos de $7200 / \mathrm{mm} 3$, neutrófilos $60 \%$, hemoglobina de $12,5 \mathrm{~g} / \mathrm{dL}$, hematocrito de $38 \%$, plaquetas de $250.000 / \mathrm{mm} 3$, proteína C reactiva de 0,7 $\mathrm{mg} / \mathrm{dL}$, velocidad de sedimentación globular $10 \mathrm{~mm} /$ hora. En el estudio ecográfico de la región escrotal se encontró una masa tumoral redondeada, con ecos mixtos, presentando áreas hipoecogénicas ubicadas encima del parénquima testicular, motivo por el que fue programado para cirugía para su extirpación, evidenciando durante la intervención que la lesión se ubica en la región del epidídimo.

En el laboratorio de patología se recibió una masa nodular quística de 3,8×2,6 cm, de color blanco amarillenta, muy bien definida que al corte era predominantemente quística con la existencia en su luz de pequeñas frondosidades papilares, friables mezcladas con un líquido de aspecto seroso. En el 
estudio de los cortes histológicos se observaba una lesión quística benigna con presencia de papilas tapizadas por epitelio cúbico bajo, de tipo seroso con amplio citoplasma claro y núcleos redondeados normocrómicos y sostenidos por estroma fibroso, congestivo de aspecto multiquístico con presencia de múltiples proyecciones papilares revestidas por epitelio seroso, con citoplasma claro, y núcleos normocrómicos, acompañada por secreción acidófila en su interior y con estroma fibroso (Ver Figura 1).

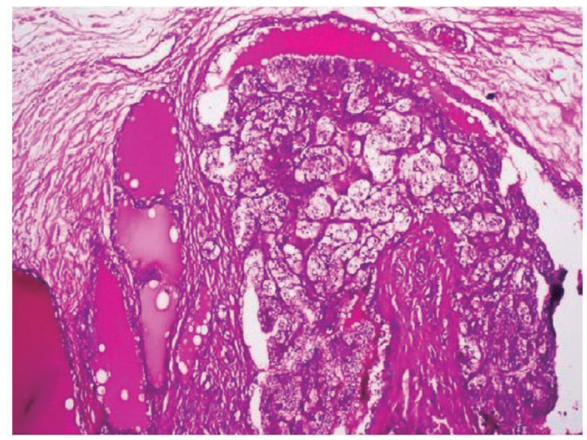

Figura 1. Se reconoce la neoplasia del epidídimo con tinción hematoxilina-eosina (10x).

Fuente: autores.

Los estudios de inmunohistoquímica revelaban que las células son reactivas para $\mathrm{CK}_{7}$ y $\mathrm{AE} 1-\mathrm{AE} 3$ y negatividad para CD10, Calretinina y CEA. Con estos hallazgos el diagnóstico es compatible con un tumor de tipo cistoadenoma seroso papilar de epidídimo (Ver Figuras 2 A y B).

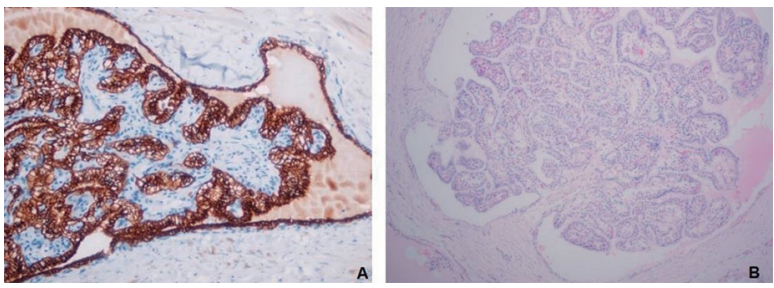

Figura 2.A. Se aprecia positividad para CK7 en la lesión quística del caso expuesto (10x). B. La lesión es negativa para el marcador CD10.

Fuente: autores.

El paciente actualmente no presenta ningún tipo de sintomatología a nivel urológico. Continúa en el proceso de rehabilitación y seguimiento por neurología, neurocirugía y fisiatría, no hay documentación de otros exámenes complementarios realizados.

\section{Discusión}

Las masas en región escrotal comprenden un amplio espectro de patologías, algunas pueden requerir diagnóstico y tratamiento temprano para evitar el riesgo de infertilidad, de pérdida del testículo o de cáncer, sobre todo en aquellos pacientes que ingresan con masas escrotales dolorosas, en la figura 3 se presenta una propuesta para el abordaje diagnóstico de un paciente con masa escrotal 7 . Se reconoce la ecografía testicular doppler como el estudio imagenológico de elección por su precisión diagnóstica, costo eficacia y gran disponibilidad, sin embargo, presenta limitaciones en sus hallazgos por su naturaleza operador-dependiente, un pequeño campo de visión que conlleva a baja visión del panorama y una pobre caracterización del tejido, se ha descrito el uso de resonancia magnética nuclear, sin embargo su uso en la práctica es muy bajo ${ }^{8,9}$. (Ver Figura 3).

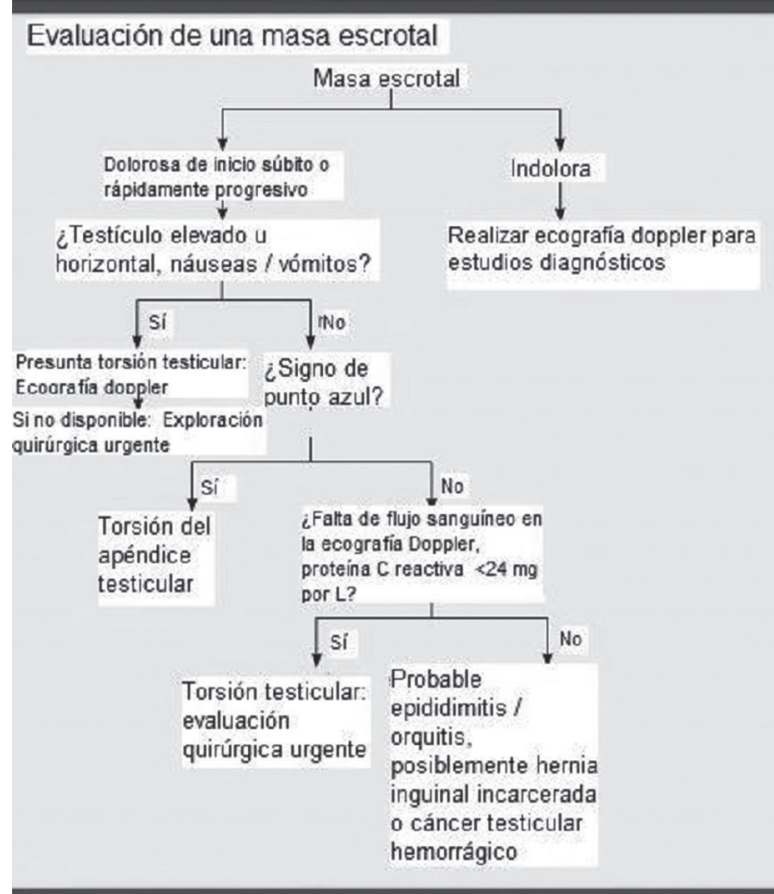

Figura 3. Algoritmo de enfoque del paciente con masa escrotal. Fuente: adaptado de referencia 7

Debe enfatizarse que dentro de las posibilidades diagnósticas de una masa escrotal se encuentran las neoplasias, en este grupo las intratesticulares corresponden en su mayoría a tumores malignos, mientras que las extratesticulares suelen ser neoplasias benignas. Un gran ejemplo de neoplasias extratesticulares, la mayoría de origen benigno, se ubican en el epidídimo².

Como se mencionó previamente, el segundo tumor benigno más frecuente localizado en el epidídimo corresponde al cistoadenoma papilar que fue 
descrito por primera vez por Lindau en 1920, más adelante otros publicaron nuevas y ampliadas descripciones microscópicas, hasta la fecha existen alrededor de 70 reportes de esta lesión con escasos datos reportados en literatura médica en castellano, y aún más limitados en países de Suramérica ${ }^{1-3,10}$. De acuerdo a lo publicado en la literatura es la primera vez que en Colombia se reporta un caso de cistoadenoma papilar de epidídimo.

Se han realizado estudios recientes que han explicado ciertos aspectos relacionados con la patogénesis de este tumor, los cuales se han basado en pacientes con enfermedad de Von Hippel Lindau y en los que se ha evidenciado lesiones precursoras confinadas a los conductos eferentes de la cabeza del epidídimo, y que, por su origen mesonéfrico es posible que los restos de material mesonéfrico mal desarrollado puedan jugar un rol importante ${ }^{4,11}$.

Macroscópicamente el cistoadenoma papilar de epidídimo se evidencia como una lesión sólida o con diferentes grados de componente quístico, típicamente menor de $5 \mathrm{~cm}$ de tamaño, con rangos que van de 0.5 hasta $8 \mathrm{~cm}$, con límites precisos, encapsulado, de color pardo amarillento, con aspecto nodular ${ }^{12-14}$. Con respecto al caso presentado, este se encuentra dentro del promedio de los descritos en la literatura con dimensiones de 3,8 $\times 2,6 \mathrm{~cm}$, además con bordes bien definidos y color blanco amarillento.

A nivel microscópico se identifican papilas con un eje conectivo vascular central cubierto por un epitelio cúbico o cilíndrico simple, compuesto por células sin criterios de malignidad, con un citoplasma claro, característica dada por la presencia de glucógeno - grasa, pocas veces estas células presentan cilios, dicho epitelio recubre ductos ectásicos y microquistes, y está acompañado de un estroma fibroso en el que es posible observar respuesta inflamatoria o cambios degenerativos. En los estudios de inmunohistoquímica la lesión suele ser positiva para marcadores epiteliales de tipo AE1/AE3, CK7 y antígeno de membrana epitelial y es negativa para CK20, CD10 y antígeno carcinoembrionario lo que va a favorecer el origen en los ductos del epidídimo 4,15 . Pueden ocurrir dificultades a la hora de realizar el diagnóstico histopatológico debido a las similitudes en las características que presenta el cistoadenoma papilar de epidídimo con el carcinoma metastásico de células renales claras, una neoplasia maligna que además también se asocia con el Síndrome de Von Hippel Lindau. Estas neoplasias comparten características morfológicas como estroma vascularizado, estructuras acinares y células claras con núcleos picnóticos centrales, e igualmente ambas se tienen origen embriológico a partir de los conductos mesonéfricos y metanéfricos ${ }^{14-16}$. En el caso expuesto los marcadores corroboran el origen en las células que revisten el epidídimo, descartando entre otros la génesis en las células mesoteliales y la presencia de lesión metastásica. Como se mencionó previamente, uno de los posibles diagnósticos diferenciales que pueden surgir es el carcinoma de células claras del riñón, dado que las células tumorales del cistoadenoma del epidídimo además de lo mencionado, tienen la capacidad de almacenar glucógeno y le dan esa apariencia de célula clara, sin embargo, como en las figuras del caso, la inmunohistoquímica realizada en el caso presentado muestra positividad para $\mathrm{CK}_{7}$ y negatividad para $C D 10$ y racemasa, mientras que para el carcinoma de células claras muestra lo contrario, es decir negatividad para CK7 y positividad para CD10 y racemasa.

De acuerdo a la literatura los casos de cistoadenoma papilar con presentación bilateral son casi patognomónicos de la enfermedad de Von Hippel Lindau pero a la fecha no existen recomendaciones precisas ni guías acerca de la conducta a seguir con estos pacientes en los que parece razonable realizar un tamizaje genético o evaluación/ seguimiento radiológico ${ }^{3,4,18}$.

La enfermedad de Von Hippel Lindau ocurre por la mutación del gen VHL, el cual está ubicado en el cromosoma 3p25-26 y actúa como un gen supresor de tumor, con herencia autosómica dominante y una penetrancia del $100 \%$ a los 65 años, por lo que la forma heredada es hasta 14 veces más frecuente que la mutación de novo que en población general corresponde a casi un 20\% de los $\operatorname{casos}^{3,18-19}$. Esta mutación otorga una predisposición a la formación de neoplasias benignas y malignas de distintos orígenes, tales como: hemangioblastomas del sistema nervioso central o retina, feocromocitoma, tumor pancreático neuroendocrino, tumor del saco endolinfático, quistes renales, quistes hepáticos, carcinoma de células claras en riñón y también cistoadenoma papilar de epidídimo o su contraparte en el sexo opuesto, el cistoadenoma papilar de ligamento ancho $0^{9,20}$. 
La relación con el Síndrome de Von Hippel Lindau es débil cuando la lesión es unilateral, por ello no se recomienda ninguna pesquisa adicional en búsqueda de este síndrome $e^{4,21-23}$. Sin embargo, recientemente se publicó un caso, hasta ahora el primero descrito, de un paciente joven de 17 años con cistoadenoma papilar de epidídimo unilateral asociado con la enfermedad de Von Hippel Lindau, el cual fue sospechado hasta un año después de la resección de la lesión escrotal, debido a hallazgos incidentales de lesiones nodulares en sistema nervioso los cuales fueron encontrados en una tomografía computarizada de cuerpo total realizada por haber presentado un accidente de tránsito 3 . El caso clínico en el presente artículo corresponde a una lesión unilateral en epidídimo, en un paciente joven de 26 años que además tiene antecedente personal de hemangioblastoma de fosa posterior documentado en el año 2011, hallazgo llamativo por su alta probabilidad de asociación con la enfermedad de Von Hippel-Lindau pero presentamos una limitación en cuanto a la confirmación diagnóstica porque por trámites administrativos no se realizaron estudios de extensión y complementarios en el paciente, pero pese a esto, continúa bajo seguimiento clínico por sus especialidades tratantes.

De igual forma, en pacientes con la enfermedad de Von Hippel Lindau que presenten cistoadenoma papilar de epidídimo uni o bilateral lo ideal sería mantener un manejo conservador, debido a que existen únicamente dos casos descritos de malignización de dichas lesiones ${ }^{23}$; teniendo en cuenta que la mayoría de los tumores extra-testiculares son benignos, es probable que el urólogo decida hacer la extracción quirúrgica de la lesión sin una confirmación histológica previa, ordenando posteriormente un estudio histopatológico ${ }^{3}$. Ante cualquier duda es posible obtener una muestra de la lesión mediante ACAF y realizar un procedimiento quirúrgico posterior según los resultados de biopsia; si bien la cirugía puede ser el tratamiento definitivo, otros autores sugieren el seguimiento de estos pacientes, al haber encontrado algunos casos de recurrencia o de transformación a cistoadenocarcinoma ${ }^{4}$. Tal y como se ha documentado en la literatura el pronóstico luego de la extirpación de la lesión es muy bueno, pero como se mencionó es esencial considerarlo como un antecedente relevante que el paciente debe recordar muy bien y que debe quedar adecuadamente registrado en la historia clínica.

\section{Conclusiones}

Las masas escrotales comprenden un amplio grupo de posibles diagnósticos que según la etiología pueden o no requerir de un manejo urgente. Dentro de los posibles diagnósticos, se encuentra el cistoadenoma papilar de epidídimo, lo más relevante de esta lesión es tener presente la posibilidad de asociarse con la enfermedad de Von Hippel Lindau, sobre todo cuando se presenta en forma bilateral. Sin embargo se debe considerar su posibilidad según el criterio del clínico en pacientes con presentación unilateral, tal como se expone en esta presentación, en la que se reporta por primera vez un caso en población del nororiente colombiano en el Hospital Universitario de Santander el cual fue confirmado con el estudio histológico y marcadores de inmunohistoquímica pues en este contexto con los antecedentes del paciente se demuestra la importancia del seguimiento multidisciplinar y de estudios de extensión por su alta probabilidad aún no demostrada de padecer la enfermedad de Von Hippel Lindau.

\section{Consideraciones éticas}

Se contó con el correspondiente consentimiento informado para el reporte del caso, así como también para el uso de los datos y hallazgos consignados en la historia clínica del paciente.

\section{Conflictos de interés}

Ninguno.

\section{Referencias bibliográficas}

1. Garrido P, Jiménez M, Herranz LM, Bocardo G, Arellano R, Pereira I. Tumor adenomatoide de epidídimo: Aportación de dos casos. Arch. Esp. Urol [Internet]. 2007 [Citado 9 Feb 2020] ;60(6):700-3. Disponible en: http://scielo.isciii.es/pdf/urol/v60n6/caso7.pdf

2. Stoisa D, Parra CM, Ferrer M, Mazzaferri FS, Villavicencio RL. Cistoadenoma papilar de epidídimo: a propósito de un caso. Rev. argent. Radiol [Internet]. 2012 [Citado 9 Feb 2020]; 76(4): 319-24. Disponible en: http://www.scielo.org.ar/pdf/rar/v76n4/v76n4a06. pdf

3. Miscia ME, Di Renzo D, Persico A, Tarallo L, Chiesa PL. Unilateral Papillary Cystadenoma of the Epididymis as a First Presentation of Von Hippel-Lindau Disease. Urology. 2018;118:189-91.

4. Odrzywolski KJ, Mukhopadhyay S. Papillary cystadenoma of the epididymis. Arch Pathol Lab Med. 2010;134:630-33.

5. Yang L, Xu WS, Melamed J, Zhou M, Deng FM. Solid variant of papillary cystadenoma of the epididymis. Histopathology. 2015;67(1):138-41.

6. Rey EP, Serramia JC. Cistoadenoma papilar de epidídimo. Actas Urol Esp. 2000;24(9):761-63. 
7. Crawford P, Crop JA. Evaluation of Scrotal Masses. Am Fam Physician. 2014;89(9):723-27.

8. Parenti GC, Feletti F, Carnevale A, Uccelli L, Giganti M. Imaging of the scrotum: beyond sonography. Insights Imaging. 2018; 9(2):137-48.

9. Tsili AC, Argyropoulou MI, Giannakis D, Sofikitis N, Tsampoulas K. MRI in the characterization and local staging of testicular neoplasms. AJR Am J Roentgenol. 2010; 194(3):682-9.

10. Cox R, Vang R, Epstein J. Papillary cystadenoma of the epididymis and broad ligament: morphologic and immunohistochemical overlap with clear cell papillary renal cell carcinoma. Am J Surg Pathol. 2014;38(5):713-18.

11. Glasker SMG, Tran SB, Shively, Ikejiri B, Lonser RR, Maxwell $\mathrm{PH}$, et al. Epididymal cystadenomas and epithelial tumourlets: effects of VHL deficiency on the human epididymis. J Pathol. 2006;210(1):32-41.

12. Soria Gondek A, Juliá Masip V, Jou Muñoz C, Salvador Hernández H, Rovira Zurriaga C, Tarrado Castellarnau X. Adolescent Hydrocele Carrying a Surprise: A Case of Papillary Cystadenoma of the Epididymis. Urology. 2018;112:172-75.

13. Gilcrease MZ, Schmidt L, Zbar B, Truong L, Rutledge M, Wheeler TM. Somatic von Hippel-Lindau mutation in clear cell papillary cystadenoma of the epididymis. Hum Pathol. 1995;26(12):134146.

14. Uppuluri S, Bhatt S, Tang P, Dogra VS. Clear cell papillary cystadenoma with sonographic and histopathologic correlation. J Ultrasound Med. 2006;25(11):1451-3.

15. Aydin H, Young RH, Ronnett BM, Epstein JI. Clear papillary cystadenoma of the epididymis and mesosalpinx: immunohistochemical differentiation from metastatic clear cell carcinoma. Am J Surg Pathol. 2005;29(4):520-3.

16. Rees S, Rashid M. Solid Variant of Papillary Cystadenoma of the Epididymis Mimicking Metastatic Clear Cell Renal Carcinoma. J Univer Surg. 2018;6(2):11.

17. Gruber MB, Healey GB, Toguri AG, Warren AG. Papillary cystadenoma of epididymis: component of von Hippel-Lindau syndrome. Urology. 1980;16(3):305-6.

18. Gatti R, Pereira MA, Giannella Neto D. Síndrome de von HippelLindau. Arq Bras Endocrinol Metab. 1999;43(5):377-88.

19. Wu P, Zhang N, Wang X, Ning X, Li T, Gong K, et al. Family history of von Hippel-Lindau disease was uncommon in Chinese patients: suggesting the higher frequency of de novo mutations in VHL gene in these patients. J Hum Genet. 2012;57(4):238-43.

20. Shen T, Zhuang Z, Gersell D, Tavassoli F. Allelic Deletion of VHL Gene Detected in Papillary Tumors of the Broad Ligament, Epididymis, and Retroperitoneum in von Hippel-Lindau Disease Patients. Int J Surg Pathol. 2000;8(3):207-12.

21. Vijayvargiya M, Jain D, Mathur SR, Iyer VK. Papillary cystadenoma of the epididymis associated with von Hippel-Lindau disease diagnosed on fine needle aspiration cytology. Cytopathology. 2014;25(4):279-81.

22. Toutziaris C, Kampantais S, Perdikis I, Gourvas V, Laskaridis L, Gkagkalidis K, et al. Papillary cystadenoma of epididymis: Is there a need for further investigation in unilateral cases?. Int J Surg Case Rep. 2013;4(7):616-18.

23. Graham L, True LD, Schweizer MT. Metastatic Adenocarcinoma of the Epididymis: A Case Report and Brief Literature Review. Clin Genitourin Cancer. 2018;16(2):e335-e38. 